\title{
Terminologia Metafórica ou Descritiva na Dermatoscopia: Qual a Melhor?
}

\author{
Nuno Gomes', Miguel Costa-Silva', Filomena Azevedo? \\ 'Departmento de Dermatovenereologia, Centro Hospitalar Universitário de São João EPE, Porto, Portugal.
}

PALAVRAS-CHAVE - Dermoscopia; Terminologia.

\section{Letter to the Editor}

\section{Metaphoric or Descriptive Terminology for Dermoscopy: Which is the Best?}

KEYWORDS - Dermoscopy; Terminology.

Em 2016, a Sociedade Internacional de Dermatoscopia (SID) publicou um documento sobre a padronização da terminologia dermatoscópica, na qual um painel de especialistas propôs um dicionário de termos quer metafóricos quer descritivos. ${ }^{1}$ Este foi um dos três marcos históricos na dermatoscopia, precedido pelo primeiro sistema de análise de padrões em 1987 e o estabelecimento de um método diagnóstico em 2003. ${ }^{2}$

A terminologia metafórica é vista por alguns autores como um aspeto natural e inevitável da linguagem humana, enquanto outros a criticam pela falta de clareza e especificidade. ${ }^{3}$ De facto, termos metafóricos como "áreas tipo folha", "dedos gordos" ou "véu azul-acizentado" são bastante subjetivos e tornam mais difícil a comunicação racional entre dermatoscopistas. ${ }^{4}$ Por outro lado, a terminologia descritiva é um conceito relativamente novo que pretende harmonizar - léxico dermatoscópico e classifica todas as estruturas pigmentadas com base em cinco elementos básicos definidos geometricamente, nomeadamente "linhas", "pseudópodes", "círculos", "glóbulos" e "pontos".

Um inquérito online anónimo questionou 1093 membros da SID sobre qual a terminologia dermatoscópica que preferiam na prática clínica diária. Um total de $56 \%$ dos participantes reportaram usar ambas as terminologias, enquanto $24 \%$ usavam preferencialmente a terminologia descritiva e $20 \%$ a terminologia metafórica. ${ }^{1}$ Giacomel J et al consideraram que a terminologia descritiva é mais apropriada para caraterizar achados dermatoscópicos simples e breves, porém torna-se de difícil aplicação quando se lida com achados complexos, nos quais termos metafóricos como "pigmentação em roda-dentada" ou "padrão em morango" podem ser eficazes. ${ }^{3}$ Independentemente das preferências pessoais, quanto mais específico for o diagnóstico evocado pela metáfora, mais útil ela se torna.

Apesar da sua utilidade prática, a linguagem metafórica pode ser uma barreira na aprendizagem da dermatoscopia. Para os mesmos achados dermatoscópicos, o dermatoscopista pode ser erradamente induzido a atribuir um diagnóstico específico. Apresentamos um exemplo da terminologia metafórica enganadora na Fig. 1 e Tabela 1. Habitualmente, somos induzidos a chamar "áreas tipo folha" a linhas radiadas castanhas conectadas a uma base comum, mas apenas se elas se apresentarem no carcinoma basocelular. $\bigcirc$ mesmo é válido para termos como "grãos de pimenta", que pode estar presente no melanoma ou na queratose liquenóide.

A importância da terminologia descritiva é salientada pelos perigosos tumores falso-negativos na dermatoscopia, nos quais neoplasias malignas que mimetizam lesões benignas podem escapar da deteção do médico. Neoplasias como - melanoma, carcinoma espinocelular e carcinoma basocelular são exemplos de lesões que podem apresentar caraterísticas de neoplasias benignas. ${ }^{5}$ É crucial adotar um método
Correspondência: Nuno Gomes Departmento de Dermatovenereologia

Centro Hospitalar Universitário de São João EPE

Alameda Professor Hernâni Monteiro

4200-319 Porto

E-mail: nunompretogomes@gmail.com

DOI: https://dx.doi.org/10.29021/spdv.78.4.1265
Recebido/Received 2020/08/19

(C) Autor (es) (ou seu (s) empregador (es)) 2020 Revista SPDV. Reutilização permitida de acordo com CC BY-NC. Nenhuma reutilização comercial.

(c) Author(s) (or their employer(s)) 2020 SPDV Journal. Re-use permitted under CC BY-NC. No commercial re-use. 


\section{Carta ao Editor}
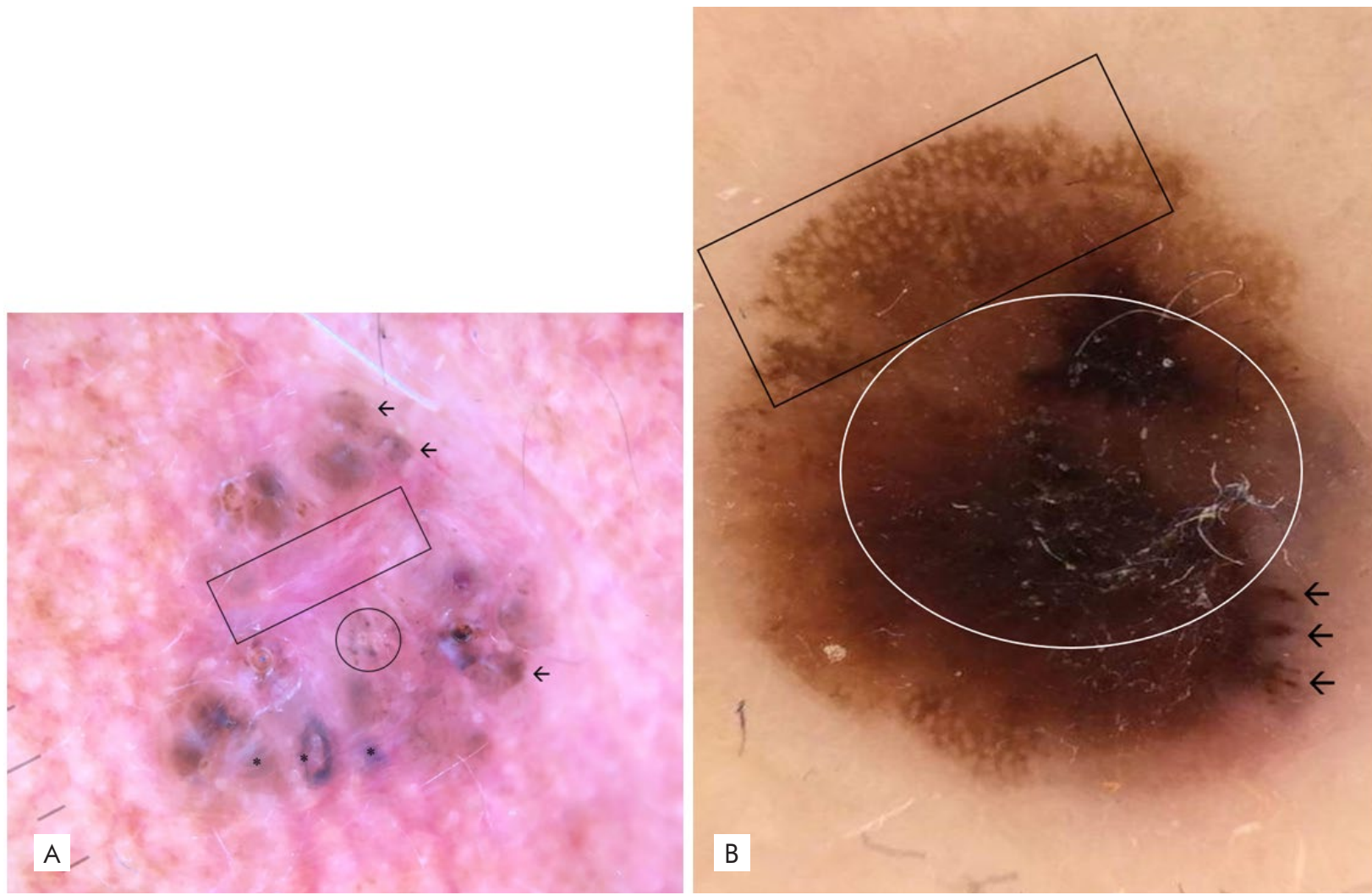

Figura 1 - (A) Dermatoscopia de contacto com luz não polarizada de uma lesão malar esquerda de um homem de 70 anos de idade, com evolução de 1 ano, cuja histopatologia confirmou carcinoma basocelular. Legenda: asteriscos - ninhos ovóides azul-acinzentados/ glóbulos grandes agrupados azuis; linhas - áreas tipo folha/ linhas radiais conectadas a uma base comum; círculos - grãos de pimenta/ pontos cinzentos; retângulo - despigmentação de tipo cicatricial/ zona sem estrutura branca. (B) Dermatoscopia de contacto com luz não polarizada de uma lesão do dorso de uma mulher de 44 anos de idade, com evolução de 2 anos, cuja histopatologia confirmou melanoma com Breslow 1,6 mm. Legenda: retângulo - rede pigmentada/ linhas reticulares; elipse - mancha/ zona sem estrutura preta; linhas - disseminação radial/ linhas radiais e segmentadas.

universal e homogéneo de terminologia dermatoscópica, evitando a dificuldade do jargão usado pelos especialistas, que pode ser incompreensível para os principiantes.
Em conclusão, a terminologia descritiva, simples e lógica, cujos termos finais não assentam num diagnóstico específico, pode ser mais adequada para aprender dermatoscopia.

Tabela 1 - Terminologia metafórica e descritiva aplicada à Figura 1.

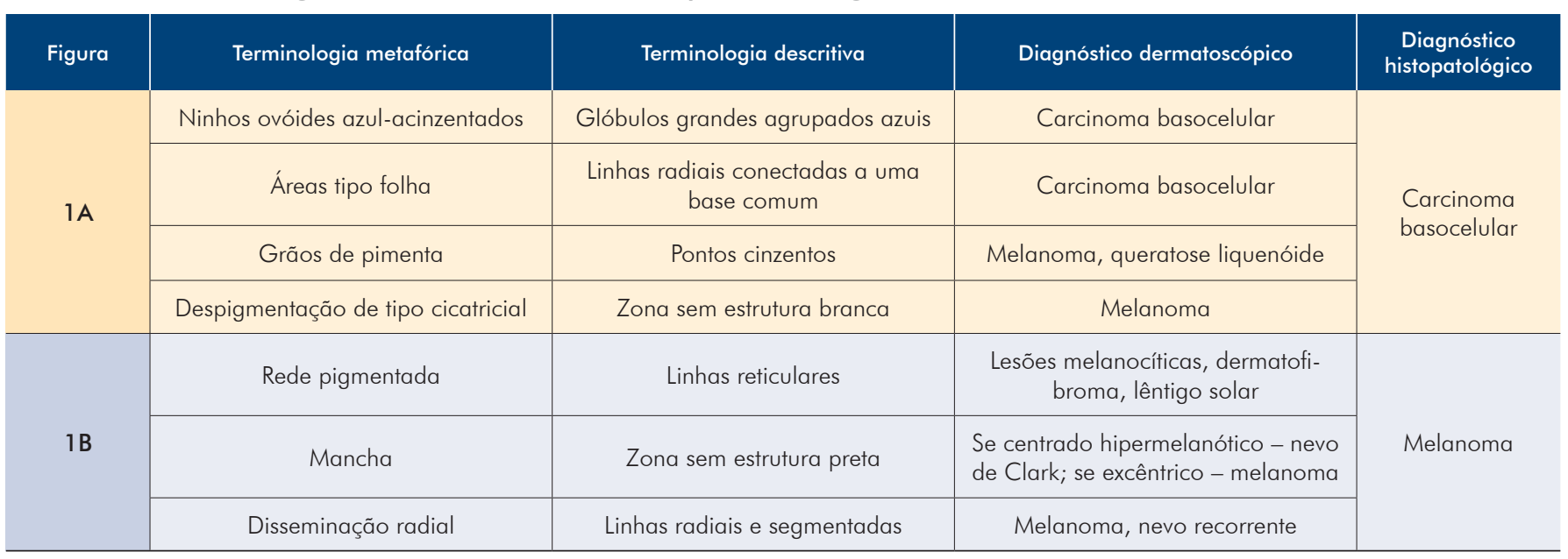


Conflitos de interesse: Os autores declaram a inexistência de conflitos de interesse na realização do presente trabalho.

Fontes de financiamento: Não existiram fontes externas de financiamento para a realização deste artigo.

Confidencialidade dos dados: Os autores declaram ter seguido os protocolos da sua instituição acerca da publicação dos dados de doentes.

Consentimento: Consentimento do doente para publicação obtido.

Proveniência e revisão por pares: Não comissionado; revisão externa por pares.

Conflicts of interest: The authors have no conflicts of interest to declare.

Financing support: This work has not received any contribution, grant or scholarship.

Confidentiality of data: The authors declare that they have followed the protocols of their work center on the publication of data from patients.

Patient Consent: Consent for publication was obtained.

Provenance and peer review: Not commissioned; externally peer reviewed

\section{ORCID}

Nuno Gomes

https://orcid.org/0000-0003-4278-3106

Miguel Costa-Silva

https://orcid.org/0000-0003-4012-5660

Filomena Azevedo

https://orcid.org/0000-0003-0402-6382

\section{Carta ao Editor}

2016;74:1093-106. doi:10.1016/j.jaad.2015.12.038.

2. Berk-Krauss J, Laird ME. What's in a Name-Dermoscopy vs Dermatoscopy. JAMA Dermatol. 2017;153:1235. doi:10.1001/jamadermatol.2017.3905.

3. Giacomel J, Zalaudek I, Marghoob AA. Metaphoric and descriptive terminology in dermoscopy: Lessons from the cognitive sciences. Dermatol Pract Concept. 2015;5:6974. doi:10.5826/dpc.0502a1 1.

4. Kittler H. Dermatoscopy: Introduction of a new algorithmic method based on pattern analysis for diagnosis of pigmented skin lesions. Dermatopathology. 2007;13:3.

5. Papageorgiou V, Apalla Z, Sotiriou E, Papageorgiou C, Lazaridou $E$, Vakirlis $S$, et al. The limitations of dermoscopy: false-positive and false-negative tumours. J Eur Acad Dermatol Venereol. 2018;32:879-88. doi:10.1111/ jidv. 14782.

\section{REFERÊNCIAS}

1. Kittler H, Marghoob AA, Argenziano G, Carrera C, Curiel-Lewandrowski C, Hofmann-Wellenhof R, et al. Standardization of terminology in dermoscopy/dermatoscopy: Results of the third consensus conference of the International Society of Dermoscopy. J Am Acad Dermatol. 